\title{
Métodos cualitativos de investigación: los grupos focales y el estudio de caso**
}

En la actualidad se experimenta el surgimiento de una forma nueva de producir el conocimiento y por consiguiente, de hacer ciencia (Gibbons, Limoges, Nowotny, Schwartzman, Scott, y Trow, 1994, pp.1-4). Esta nueva forma se caracteriza por operar en un contexto en el que los problemas de investigación no son dictados por una disciplina en particular, sino que emergen de las experiencias cotidianas. Es decir, del mundo del trabajo, de las organizaciones y de las relaciones sociales. Además, es transdisciplinaria en lugar de mono o multidisciplinaria; es heterogénea; es multiforme y temporal en lugar de jerárquica y permanente; es más responsable socialmente y reflexiva que distanciada de su entorno.

Esta nueva forma de hacer ciencia no se limita a una metodología positivista de investigación, sino que incorpora los métodos cualitativos desarrollados originalmente en la antropología (Malinosky, 1922) y la sociología (Douglas, 1970). Posteriormente estos métodos fueron incorporados a la etnometodología (Garfinkel, 1975, p.18) en el estudio de las culturas y a la etnografía en escenarios organizacionales (Schwartzman, 1993, p.2).

Por método cualitativo debe entenderse cualquier tipo de investigación que no depende de medios de cuantificación o procedimientos estadísticos para generar sus hallazgos (Strauss y Corbin, 1990, p.17). Por ejemplo, si estuviésemos interesados en investigar la satisfacción en el trabajo resultaría valioso conocer el número de empleados satisfechos, el

Las investigaciones de orientación cualitativa representan una nueva forma de pensar y de hacer ciencia. En la actualidad se han articulado en un paradigma de orientación fenomenológica que asume una perspectiva multicultural y la aceptación de múltiples realidades. Sus hallazgos constituyen una formulación teórica sobre la realidad bajo estudio. En lugar de aspirar a reunir un conjunto de números o un grupo de temas, ligeramente relacionados, sus métodos aspiran a construir una teoría que ilumine y sea fiel al área sustantiva de estudio.

*Sicólogo Industrial, Consultor de Reyes \& Asociados y Profesor de Sicología Industrial en la Facultad de Ciencias Sociales.

** Conferencia presentada en la Facultad de Administración de Empresas de la Universidad de Puerto Rico, Recinto de Río Piedras por invitación del Centro de Investigaciones Comerciales e Iniciativas Académicas en marzo 1998. Revisada en enero de 2000 . 
grado de satisfacción que exhiben y probablemente la correlación entre el grado de satisfacción y los factores que la producen; pero ningún número o procedimiento estadístico permite identificar cuáles son los factores que promueven la satisfacción o el porqué unos factores generan mayor satisfacción que otros.

Así también ocurre en la investigación sobre los problemas sociales. En el caso de la adicción a drogas, por ejemplo, resulta valioso conocer el número de adictos, el número de adictos por sustancia, la frecuencia del consumo, el costo del consumo y probablemente la correlación entre el consumo de sustancias y la comisión de delitos. Ello permitiría establecer prioridades para el tratamiento e intervención que correspondan a la magnitud del problema, pero ninguno de esos datos ofrece información sobre las causas del comportamiento adictivo, ni explica las diferencias en la preferencia de una sustancia sobre otra o la relación entre el tipo de sustancia y las características de un particular tipo de consumidor.

En estos ejemplos se puede ver cómo tanto la investigación cuantitativa como la cualitativa, contribuyen al éxito del quehacer científico. La decisión de usar uno u otro enfoque probablemente radica en la preferencia del investigador por una metodología en particular, en la formación del investigador, o en la naturaleza del problema. Algunos fenómenos se prestan mejor a un abordaje cualitativo. La investigación cualitativa puede ser útil en el descubrimiento y la comprensión de aquellos asuntos que subyacen cualquier fenómeno desconocido. También para lograr ópticas nuevas o para poner al descubierto los detalles de cualquier fenómeno, en el estudio de las vidas de las personas, sus historias y sus comportamientos; así como también el funcionamiento de las organizaciones, los movimientos sociales o las relaciones interpersonales.

En la actualidad, los métodos cualitativos se han articulado en un paradigma de orientación fenomenológica que asume una perspectiva multicultural y la aceptación de múltiples realidades. Según Fetterman (1989, p.15) las personas actúan guiadas por sus percepciones individuales y tales acciones tienen consecuencias reales. Por consiguiente, la realidad subjetiva que cada individuo ve, no es menos real que una definida y medida objetivamente. El investigador cualitativo está interesado en entender y describir una escena social y cultural desde adentro, desde la perspectiva del que la vive. Mientras mejor entienda el investigador el punto de vista del actor social, mejor la descripción y mejor la ciencia. En esa gestión, el investigador se convierte en una especie de científico y narrador al mismo tiempo.
La investigación cualitativa es un método y un arte para describir un grupo o una cultura. La tarea del científico es similar a la de un periodista que entrevista a personas reconocidas, revisa récords, considera la opinión de una persona sobre la opinión de otra, busca los lazos entre intereses especiales y las organizaciones, y escribe la historia para un público concernido así como también para sus colegas profesionales.

A diferencia del periodista, quien estudia y escribe sobre lo sensacional y lo poco usual, el investigador cualitativo estudia los asuntos cotidianos. Aquellos patrones del comportamiento y del pensamiento humano que ocurren en el día a día. Los investigadores cualitativos son reconocidos por su capacidad para mantener una mente abierta ante los grupos o culturas que estudian, sin que ello implique falta de rigor científico. Esto no quiere decir que el investigador cualitativo se aproxima a su objeto de estudio sin unas bases conceptuales pensadas. Se dice que los investigadores cualitativos se incertan en el campo de estudio con una mente abierta, no con una mente vacía.

Esa capacidad de mantener una mente abierta le permite al investigador explorar fuentes de datos que no fueron consideradas al momento de diseñar el estudio. Más aún, la investigación cualitativa permite múltiples interpretaciones de la realidad, así como interpretaciones que pueden variar a través del tiempo.

Es un enfoque particularmente valioso porque problematiza las formas en las que los individuos y los grupos constituyen e interpretan las organizaciones y sociedades. Además, facilita el aprendizaje de las culturas y las estructuras organizacionales mediante el examen del conocimiento, el comportamiento y las maneras que los investigados usan para interpretar sus experiencias (Schwartzman, 1993, p.4, Jacob 1987, 1988) ha categorizado las diversas tradiciones en la investigación cualitativa en seis orientaciones principales: la etología humana, la psicología ecológica, la etnografía holística, la antropología cognitiva, la etnografía de la comunicación y el interaccionismo simbólico.

1. La etología humana busca entender la extensión del comportamiento humano. Los datos son recopilados mediante la observación y analizados cuantitativamente.

2. La psicología ecológica estudia la interacción de las personas y el ambiente en la producción del comportamiento. Descansa en la observación suplementada con récords. 
3. La etnografía holística busca entender el rol de la cultura mediante el empleo de la observación partícipe. Los etnógrafos tratan de descubrir y documentar la perspectiva de los participantes.

4. La antropología cognitiva asume que la perspectiva de los participantes está organizada en categorías cognoscitivas o semánticas que le imprimen significado y que están sistemáticamente relacionadas las unas con las otras.

5. La etnografía de la comunicación se centra en datos sobre interacciones verbales y no verbales, descansando en la observación de los participantes y la grabación audiovisual de dichas interacciones.

6. En el interaccionismo simbólico el interés es entender cómo los individuos le dan significado a su interacción con otros. El énfasis es en el proceso de significación.

En cada una de estas orientaciones las destrezas que necesita un investigador son: la sensibilidad teórica y social, la habilidad para mantener distancia analítica, las destrezas de observación y las destrezas de interacción social. Según Strauss \& Corbin (1990, p. 18), el investigador cualitativo debe ser "conocedor de los datos y la teoría y al mismo tiempo capaz de escapar los aspectos de su propio trabajo que puedan bloquear la nueva perspectiva, el presentimiento, la intuición, la idea brillante, o la formulación teórica diferente."

\section{Validez y confiabilidad}

En las ciencias la validez y la confiabilidad son los criterios mediante los cuales se puede evaluar una particular metodología. En general, el quehacer científico se ha caracterizado por un discurso en el cual la validez es vista como un ingrediente tangible de la que si uno puede adquirir una cantidad suficiente, ha ganado en el juego llamado investigación científica. Particularmente si dicho ingrediente es adquirido mediante la aplicación de técnicas "apropiadas". Un análisis crítico de la literatura disponible arroja dos conclusiones fundamentales sobre el concepto de validez. En primer lugar, la validez es una variable hipotética de naturaleza correlacional. Esto es, la validez es un atributo inferido de la correlación que pueda obtenerse entre variables. Por ejemplo, las puntuaciones de los reactivos de una prueba y otros criterios como la puntuación total de la prueba, las puntuaciones de otras pruebas y alguna otra puntuación que represente un criterio o una conducta.

En segundo lugar, la validez no es absoluta, sino que está determinada por el contexto situacional y el uso que se le dará a la prueba o a sus resultados (Cirino, 1984, p.195; Cronbach, 1960, p.96). En este sentido, más que con un número, la validez tiene que ver con la veracidad y con el valor del trabajo científico. La validez al igual que el carácter o la calidad, debe ser determinada en relación al propósito y las circunstancias en las que se produce (Brinberg y McGrath, 1985, p.13).

El otro criterio clave de toda gestión científica es la confiabilidad. En el marco positivista la confiabilidad es expresada en términos de un coeficiente de correlación, como la relación entre dos medidas obtenidas de la misma forma o como el error estándar de la medición. Según Anastasi (1988, p.109), en su sentido más amplio la confiabilidad indica el grado en el cual las diferencias individuales pueden atribuirse a diferencias verdaderas en las características bajo estudio y el grado en que pueden atribuirse a errores de medición.

El problema con dichas atribuciones es que a pesar de ser construcciones conceptuales, están representadas por datos numéricos que no resultan útiles para conocer la naturaleza de las diferencias individuales observadas, ni a qué variables está asociado el error en la medición. Kirk y Miller (1986, pp.41-42), distinguen tres tipos de confiabilidad: la quijotesca, la diacrónica y la sincrónica.

1. La confiabilidad quijotesca se refiere a la confiabilidad que se obtiene mediante un solo método de observación que arroja una medida que no varía a través del tiempo.

2. La confiabilidad diacrónica se refiere a la estabilidad de una observación a través del tiempo.

3. La confiabilidad sincrónica se refiere a la similaridad de las observaciones dentro de un mismo periodo de tiempo.

En el marco de la investigación cualitativa, la confiabilidad es la probabilidad de obtener los mismos resultados dentro de un mismo periodo de tiempo o resultados relacionados a través del tiempo. La diferencia entre la confiabilidad cuantitativa y la cualitativa es que en la primera, la confianza está puesta en un número; mientras que en la segunda, la confianza reside en la apreciación del binomio investigado/investigador. 
La validez y la confiabilidad no son sustancias que uno pueda adquirir mediante el uso de técnicas particulares, sino conceptos que designan un estado al que debe aspirar todo esfuerzo por descubrir y producir conocimiento.

\section{Metodología cualitativa}

Por lo general en el proceso de investigación cualitativa se observan los siguientes pasos: el diseño del estudio, la recopilación de datos, el análisis de datos y la retroinformación (Marshall \& Rossman, 1995). El diseño consiste en formular las preguntas de investigación considerando el uso que habrá de hacerse de los resultados, el interés puramente científico de adelantar el conocimiento o la petición específica de un cliente.

El diseño también incluye la selección de la muestra de estudio y de las técnicas de investigación a usarse. Es decir, las técnicas a emplearse en la recopilación de los datos. Tanto la fuente de la petición como la naturaleza de la investigación inciden en el tipo de muestra, así como en las técnicas de investigación a seleccionarse. Además, el diseño considera toda la logística de la investigación como los materiales, el equipo, la duración, los recursos y el costo del proyecto.

La recopilación de datos consiste en las actividades desarrolladas y la aplicación de las técnicas de investigación seleccionadas para recoger la información. La investigación cualitativa siempre se inicia con la inserción del investigador en el entorno a estudiarse. Tanto en comunidades, como en empresas u otras instituciones, la inserción es un proceso crítico que tiene que ser negociado entre el investigador y el investigado.

El análisis de los datos consiste en la aplicación de diversos procedimientos para organizar e interpretar la información de forma tal que arroje un retrato fiel de la realidad según la vive el investigado. Entre los procedimientos utilizados está el análisis de contenido, el análisis semántico y el uso de estadísticas descriptivas. Cuando la investigación ha sido solicitada para resolver algún problema particular de una organización, el análisis de los datos es sumamente crítico porque en ocasiones los hallazgos pueden estar en conflicto con la política o el estilo administrativo de la empresa.

La retroinformación se refiere a la forma seleccionada para trasmitir los resultados de la investigación. Entre las formas frecuentemente utilizadas se encuentra el informe de investigación, la presentación en foros concernidos, y el diseño de intervenciones destinadas a atender el problema o necesidad que inició la investigación.

Cuando la investigación cualitativa está ligada a problemas o necesidades específicas, el científico se convierte en una especie de investigador/ consultor que intenta obtener resultados aplicables a situaciones reales. En este sentido, la calidad de la investigación no se mide por su adecuación a un modo particular de ver la ciencia, sino por la contribución que hace a la solución del problema de interés.

Con frecuencia se utilizan los términos "método de investigación" y "técnicas de investigación" como sinónimos. En ocasiones este uso ha conducido a algunos a confundir los procedimientos estadísticos con el paradigma cuantitativo y viceversa. Un método es un concepto que designa la adecuacidad entre la actividad de investigar y las bases teóricas que sustentan la investigación. El método establece los parámetros que han de guiar la pesquisa, mientras que una técnica de investigación es la herramienta que se utiliza para recopilar los datos (Olson, 2000). Por ejemplo, un experimento o un estudio de campo son métodos de investigación porque delimitan las maneras de acercarse al objeto de estudio (la manipulación de variables, en el caso del experimento, y la naturalidad del escenario en el caso del estudio de campo), pero un cuestionario o una entrevista son técnicas porque sólo sirven para recoger información.

El método de investigación cualitativa se caracteriza por integrar una variedad de técnicas. Una de las iniciales fue la entrevista no directiva en la que, contrario a la directiva, el entrevistado tenía un rol activo. El énfasis reside en alinearse con la realidad del entrevistado (Strauss \& Corbin,1990). Los procedimientos se inician con un conjunto limitado de supuestos, utilizan preguntas abiertas y se realizan en un ambiente que le permite a los participantes contestar sin límites ni claves (Krueger, 1991, p.19). Contrario a la entrevista directiva, los acercamientos abiertos le ofrecen al investigado una oportunidad amplia para comentar, para explicar y compartir sus experiencias y sus actitudes (Krueger, 1991, p.19).

Entre las técnicas de investigación cualitativa conocidas están las siguientes:

- La Observación Partícipe

- La Entrevista

- Los Informantes Claves

- Los Grupos Focales
- Las Biografías

- La Revisión de Documentos Oficiales

- El Estudio de Caso

- Los Cuestionarios 
Cada técnica tiene la capacidad de modificarse y ajustarse a las necesidades de la investigación. Por ejemplo, la entrevista puede ser grupal, individual, grabada, filmada o escrita. A continuación se discutirán dos de las técnicas utilizadas con frecuencia: el grupo focal y el estudio de caso.

\section{El grupo focal}

Un grupo focal puede definirse como una discusión cuidadosamente diseñada para obtener las percepciones de una muestra sobre un área particular de interés (Krueger, 1991, p.18). Los grupos focales están enraizados en una tradición desarrollada a partir de 1930 que intentaba conocer los mundos a través de las personas que los vivían. Algunas de las áreas que se han beneficiado del uso de los grupos focales son la Planificación Estratégica, la Identificación de Necesidades, y la Evaluación de Programas.

Para los profesionales que quieren mejorar los programas y la prestación de servicios, los grupos focales permiten obtener información sobre las percepciones, los sentimientos, y las actitudes de los clientes sobre sus programas (Krueger, 1991). La capacidad de una empresa para enfocarse en el cliente puede hacer la diferencia entre mantenerse y trecer o desaparecer del mercado. Por ejemplo, los autores Peters y Waterman (1982, pp. 170171) atribuyen parte de la excelencia de las industrias norteamericanas a su capacidad para mantenerse enfocadas en los clientes.

Según Krueger (1991, p.23), la entrevista de grupo focal funciona porque incide en las tendencias humanas. Las actitudes y las percepciones relacionadas con los productos, servicios o programas, son desarrolladas en parte mediante la interacción entre las personas. La evidencia sugiere que las opiniones de un individuo pueden cambiar en el curso de la discusión con otros porque las personas se influencian las unas a las otras por medio de sus comentarios. Los analistas pueden descubrir información sobre cómo dicho cambio ocurrió y la naturaleza de los factores influyentes.

El objetivo de un grupo focal es promover la apertura de los participantes. Para algunos individuos, la autoexposición les resulta fácil, natural y cómoda, para otros difícil e incómoda porque la auto-exposición requiere confianza, esfuerzo y coraje (Krueger, 1991, p.23). Estos grupos se caracterizan por estar constituidos por personas que poseen ciertas características en común. Estas personas proveen datos o información de naturaleza cualitativa mediante su participación en una discusión enfocada (Krueger, 1991, p.44). Los grupos deben ser lo suficientemente pequeños como para permitir la oportunidad a cada participante de compartir su conocimiento y a la vez lo suficientemente grandes como para proveer una diversidad de percepciones sobre el asunto de interés.

Los procedimientos cualitativos como los grupos focales o las entrevistas individuales, capacitan al investigador para alinearse con el participante y descubrir cómo la persona ve la realidad. Al igual que otros procedimientos de las Ciencias Sociales, la validez de éstos depende de los procedimientos usados y del contexto donde son usados. De acuerdo con Krueger (1991) entre sus ventajas están las siguientes:

- Son socialmente orientados y los participantes interactúan en situaciones reales y naturales versus las condiciones rígidamente estructuradas de los experimentos.

- El formato de las discusiones le ofrece al facilitador o moderador la flexibilidad necesaria para explorar asuntos que no hayan sido anticipados.

- Poseen validez aparente ("face validity"), la técnica es fácil de entender y los resultados son creíbles para los usuarios de la información.

- El costo de las discusiones es relativamente bajo.

- Son ágiles en la producción de resultados.

- Le permiten al investigador aumentar la muestra de estudio sin aumentar dramáticamente el tiempo de investigación.

En Puerto Rico, el uso de los grupos focales se ha limitado principalmente al sector de la venta y la publicidad (Entrevista personal, marzo de 1998). En algunas excepciones, los grupos focales han sido utilizados para diseñar intervenciones sociales. Por ejemplo, en 1991 la información sobre las causas de la deserción escolar obtenida mediante el uso de grupos focales fue utilizada para diseñar un programa de prevención (Reyes, 1991). La efectividad del programa se debió, en gran parte, a la precisión con que se identificaron los elementos de riesgo. Es decir, los generadores de la deserción.

\section{El estudio de caso}

El estudio de caso es un examen intensivo a una entidad de una categoría o especie. Esta técnica permite la recopilación e interpretación detallada de toda la información posible sobre un individuo, una sola institución, 
una empresa, o un movimiento social particular. Puede hacer uso de pruebas en las que se emplean preguntas diseñadas para conocer la entidad bajo estudio. Más aún, cuando se emplea como técnica de investigación la meta no consiste únicamente en conocer la entidad a la que se estudia, sino también conocer la categoría que representa.

Los estudios de caso pueden clasificarse en dos tipos: el primero intenta derivar conclusiones generales a partir de un número limitado de casos; el segundo tipo intenta llegar a conclusiones a partir de un solo caso debido a la importancia o interés particular de su historia. Yin (1984), distingue tres usos de esta técnica: el exploratorio, cuyos resultados pueden ser usados como base para formular preguntas de investigación más precisas o hipótesis que puedan ser probadas; el descriptivo, que intenta reseñar lo que sucede cuando un producto nuevo es desarrollado o lanzado al mercado; y el explicativo, que facilita la interpretación de las estrategias y procesos de trabajo que utiliza una compañía en particular. Este último tipo es sumamente útil para generar teorías, así como para iniciar cambios en una organización.

Las observaciones detalladas del estudio de caso permiten estudiar múltiples y variados aspectos con un mismo objeto de examen. Además, permiten examinar cada uno de esos aspectos en relación con otros dentro de su ambiente total. Esta capacidad de la técnica resulta en una de sus principales ventajas: la oportunidad que ofrece para desarrollar una visión holística del objeto de estudio.

Otra de las ventajas, y tal vez la más importante, es la capacidad que ofrece para aplicar sus resultados. La familiaridad de los gerentes con el lenguaje, los datos y el análisis facilitan el diseño de cualquier intervención. Más aún, la riqueza conceptual y descriptiva de los datos le permite al investigador determinar la aplicabilidad de los hallazgos a sus particulares circunstancias. Según Myers (2000) entre las críticas principales señaladas a estos estudios se encuentran las siguientes:

- que no tienen validez estadística,

- que pueden servir para generar teorías, pero no para probarlas y

- que no permiten hacer generalizaciones.

Si bien estas críticas parecen lógicas, es sólo si se miran a través del prisma positivista. Como hemos señalado, la validez científica no descansa en el establecimiento de una correlación, sino en la comprensión fundamental de la estructura, los procesos y las fuerzas que mueven al ente de estudio.
La capacidad del investigador para adquirir el conocimiento fundamental sobre la organización y sus actores sociales, no descansa en su habilidad para establecer relaciones de causa y efecto, sino en su habilidad para desarrollar un lenguaje común y conceptos apropiados al caso bajo estudio.

Finalmente, la capacidad de generalizar a partir de un solo caso no reside en el tamaño de la muestra, sino en la calidad de las medidas y los datos que se recogen (Gummesson, 1991, 78-79). En la investigación cualitativa la generalización depende de cuán abarcadoras sean las medidas que se tomen y el rigor científico del proceso de descubrimiento.

\section{Conclusión}

Los métodos cualitativos aumentan las alternativas válidas y confiables de investigación disponibles. Esta aproximación metodológica valora las perspectivas que los propios investigados tienen sobre sus mundos y busca descubrir esas perspectivas. El carácter de ciencia a estos métodos se lo imprime la compatibilidad entre la teoría y la observación, su capacidad para replicar el estudio, el grado en que se pueden generalizar sus conclusiones y la precisión y el rigor de su abordaje al objetivo de estudio.

Estos métodos requieren la inmersión del investigador en la vida cotidiana de los participantes, reconocen la relación de reciprocidad entre el investigador y el investigado, son principalmente descriptivos y dependen del lenguaje y de los comportamientos de los actores sociales como fuentes de datos primarios.

Una de las técnicas discutidas, los grupos focales, le permite al investigador alinearse con el participante y descubrir cómo las personas ven la realidad. La validez de esta técnica depende de los procedimientos utilizados y del contexto donde se implanten sus resultados.

Por su parte el estudio de caso permite examinar múltiples aspectos de un solo individuo, una organización, una comunidad o un sector del mercado. La capacidad para generalizar a partir de un solo caso es limitada y depende de la calidad de los datos.

Finalmente, las investigaciones de orientación cualitativa representan una nueva forma de pensar y de hacer ciencia. Sus hallazgos constituyen una formulación teórica sobre la realidad bajo estudio. En lugar de aspirar a reunir un conjunto de números o un grupo de temas ligeramente relacionados, sus métodos aspiran a construir una teoría que ilumine y sea fiel al área sustantiva de estudio. 


\section{Referencia}

Anastasi, A. (1988). Psychological Testing. New York: MacMillan Publishing Company.

Brinberg, D. \& McGrath, J.E. (1985). Validity and the Research Process. Beverly Hills; California: Sage.

Cirino, Gerena G. (1984). Introducción al desarrollo de pruebas escritas. Río Piedras: Editorial Bohío.

Cronbach, L. J. (1960). Essentials of Psychological Testing. New York: Harper \& Row.

Douglas, J. D. (1970). Understanding Everyday Life. Chicago: Aldine.

Entrevista personal: Begoña Robledo, Vicepresidenta de Hispania Research Corporation, marzo de 1998.

Fetterman, D.M. (1989). Ethnography Step by Step. Beverly Hills; California: Sage.

Garfinkel, H. (1975). The Origins of the Term Ethnomethodology. En R, Turner (Ed.) Ethnography: Selected Readings. Middlesex, England: Penguin.

Gibbons, M.; Limoges, C.; Nowotny, H.; Schwartzman, S.; Scott, P. \& Trow, M. (1994). The New Production of Knowledge: The Dynamics of Science and Research in Contemporary Societies. Beverly Hills; California: Sage.

Gummesson, E. (1991). Qualitative Methods in Management Research. Beverly Hills; California: Sage.

Jacob, E. (1987). The Traditions of Qualitative Research: A Review. Review of Educational Research, 51, 1-50.

Jacob, E. (1988). Clarifying Qualitative Research: A Focus on Traditions. Educational Researcher, 17, 16-24.

Kirk, J. \& Miller, M.L. (1986). Reliability and Validity in Qualitative Research. Beverly Hills; California: Sage.

Krueger, R.A. (1991). Focus Groups: A Practical Guide for Applied Research. Beverly Hills; California: Sage.

Lincoln, Y.; Guba, E. (1985). Naturalistic Inquiry. Beverly Hills; California: Sage.
Malinosky, B. (1922). Argonauts of the Western Pacific. London: Routledge.

Marshall, C.; Rossman, G.B. (1995). Designing Qualitative Research. Beverly Hills; California: Sage.

Myers, M. (2000). Qualitative Research and the Generalizability Question: Standing Firm with Proteus. The Qualitative Report, 4, 3/4, Marzo 2000. http:// www.nova.edu/ssss/QR/QR4 -3/ myers.bhtml.

Olson, H. (2000). Quantitative "Versus" Qualitative Research: The Wrong Question. http://www.ualberta.ca/dept/slis/cais/olson.htm

Peters, T. J.; Waterman, R.J. (1982). In Search of Excellence. New York: Harper \& Row.

Reyes, T. (1991). Retos: Retención escolar, trabajo y ocupaciones. Programa de Adiestramiento para el Empleo, Municipio de San Juan.

Schwartzman, H.B. (1993). Ethnography in Organizations. Beverly Hills; California: Sage.

Strauss \& Corbin, (1990). Basic Qualitative Research: Grounded Theory Procedures and Techniques. Beverly Hills; California: Sage.

Yin, R. K. (1984). Case Study Research. Beverly Hills; California: Sage. 